\title{
Psychological and Environmental Correlates of HPA Axis Functioning in Parentally Bereaved Children: Preliminary Findings
}

\author{
Julie B. Kaplow, ${ }^{1}$ Danielle N. Shapiro, ${ }^{2}$ Britney M. Wardecker, ${ }^{2}$ Kathryn H. Howell, ${ }^{1}$ James L. Abelson, ${ }^{1}$ \\ Carol M. Worthman, ${ }^{3}$ and Alan R. Prossin ${ }^{1}$ \\ ${ }^{1}$ Department of Psychiatry, University of Michigan Medical School, Ann Arbor, Michigan, USA \\ ${ }^{2}$ Department of Psychology, University of Michigan, Ann Arbor, Michigan, USA \\ ${ }^{3}$ Department of Anthropology, Emory University, Atlanta, Georgia, USA
}

\begin{abstract}
This study examined bereaved children's HPA-axis functioning (cortisol awakening response; CAR) in relation to psychological distress, coping, and surviving parents' grief reactions. Participants included 38 children (20 girls) with recent parental loss (previous 6 months) and 28 of their surviving caregivers ( 23 women) who were assessed using self-report instruments and in-person, semistructured interviews. Interviews involved discussions about the child's thoughts and feelings related to the loss. Participants provided 3 saliva samples at home (awakening, 30 minutes later, and evening) over 3 successive days, beginning on the day following the interview. Results show a significant relation between dampening of the child's Day 1 CAR and more symptoms of anxiety $(r=-.45)$, depression $(r=-.40)$, posttraumatic stress $(r=-.45)$, and maladaptive grief $(r=-.43)$, as well as higher levels of avoidant coping $(r=-.53)$. Higher levels of parental maladaptive grief were also associated $(r=-.47)$ with a dampening of the child's Day 1 CAR. Our results raise the possibility that blunted CAR may be a result of accumulating allostatic load and/or a result of emotionally challenging events (discussions regarding the deceased) and their subsequent processing (or lack thereof) within the family, which may be particularly stressful for those bereaved children experiencing high levels of psychological distress, avoidant coping, and parental maladaptive grief.
\end{abstract}

Parental loss during childhood is a significant public health problem, with the most recent projected estimate of 25 million newly orphaned youth worldwide in 2010 alone (UNAIDS, UNICEF, \& USAID, 2004). Symptoms of distress are evident in parentally bereaved children (Goenjian et al., 2009; Kaplow, Saunders, Angold, \& Costello, 2010), and a significant minority (approximately 10\%; Dowdney, 2000; Melhem, Porta, Shamseddeen, Walker Payne, \& Brent, 2011) exhibits clinically disruptive grief reactions. The biological, psychological, and environmental predictors of such reactions are still largely

This research was supported in part by grants NIMH K08 MH76078, the Todd Ouida Clinical Scholars Award, and the Laurence Polatsch Memorial Fund given to the first author. The authors wish to thank Michelle Belke, Alena Grieser, Heather Hammerstrom, Harriett Jones, Georgia Stamatopoulos, and Amanda Tarantowski for their assistance with conducting interviews. We also thank Emilie Lerner for her assistance with project coordination and Valerie Elsesser, Kara Koppinger, Mirele Mann, Meredith Merlanti, and Teera Parr for their assistance with data management, data entry, and cortisol assays. Finally, we wish to acknowledge the Gilda's Club of Grand Rapids staff for their administrative support, and all participating children, parents, and guardians.

Correspondence concerning this article should be addressed to Julie B. Kaplow, Department of Psychiatry, University of Michigan, Rachel Upjohn Building, 4250 Plymouth Road, Ann Arbor, MI 48109-5765. E-mail: julieb@med.umich.edu

Copyright () 2013 International Society for Traumatic Stress Studies. View this article online at wileyonlinelibrary.com

DOI: $10.1002 /$ jts. 21788 unknown. In the midst of ongoing developmental changes, bereaved children depend heavily on their immediate caretaking environment to help facilitate their mourning (Clark, Pynoos, \& Goebel, 1994). Accordingly, efforts to distinguish between features of positive adjustment versus maladjustment following bereavement in youth must address grief and mourning within the broader context of both individual (e.g., biological mechanisms, coping strategies) and socioenvironmental (e.g., parental psychopathology) factors that may diminish, promote, or serve as markers of these outcomes (Kaplow, Layne, Pynoos, Cohen, \& Lieberman, 2012). The identification of biological and psychosocial mechanisms linking childhood bereavement to psychiatric symptoms will help to inform the development of targeted interventions for at-risk bereaved youth.

The hypothalamic-pituitary-adrenal (HPA) axis and its end product, cortisol, play a key role in mediating the negative impact of stress on the onset, course, and pathophysiology of psychiatric disorders (Adam \& Kumari, 2009; Pfeffer, Altemus, Heo, \& Jiang, 2007) and could serve as a mediator of or risk marker for maladaptive responses to early parental loss. Short-term elevations in cortisol are critical for survival under stressful circumstances, but prolonged exposure to high cortisol levels appears to be associated with serious health risks (Bevans, Cerbone, \& Overstreet, 2008). Hypercortisolemia or impaired feedback control of the HPA axis, for example, are well-established biomarkers of depression, in both adults 
(e.g., Gold, Goodwin, \& Chrousos, 1988; Young, Haskett, Murphy-Weinberg, Watson, \& Akil, 1991) and children/ adolescents (e.g., Kaufman et al., 1997; Rao et al., 1996). A growing body of evidence, however, suggests that blunted reactivity in this system also may play a role in the pathophysiology of stress-related disorders (for review see Gunnar \& Vasquez, 2001).

"Hypocortisolism" can be characterized by low cortisol secretion, flat cortisol production patterns during the day, or blunted cortisol responses to stressors (Heim, Ehlert, \& Hellhammer, 2000); all of these have been associated with exposure to early life stress. Early life adversity may provoke frequent elevations in cortisol that lead to compensatory downregulation and reduced activity of the HPA axis in later life (Gunnar \& Vasquez, 2001). Others (e.g., Hellhammer \& Wade, 1993; McEwen 1998) have similarly argued that hypocortisolism can reflect allostatic load, defined as the cumulative burden and physiological consequences of chronic exposure to heightened neuroendocrine response that results from chronic stress. For example, a history of repeated trauma exposure in youth (higher levels of both previous and recent trauma, including the death of a loved one) is associated with blunted morning cortisol levels (Bevans et al., 2008). A poorly reactive stress system, perhaps created by early adversity exposure, may then contribute to psychiatric vulnerability. Consistent with this view, lower morning cortisol levels and a blunted cortisol response have been associated with poor parental responsiveness, a neglectful environment, and insecure attachment patterns (Carlson \& Earls, 1997; Spangler \& Grossman, 1993) during infancy and early childhood, all of which may contribute to psychopathology risk.

Efforts to link HPA axis dysfunction directly to psychiatric symptoms in youth have produced variable results. High cortisol levels were positively associated with PTSD symptoms in trauma-exposed children, but only when the trauma was recent (past year); with prior trauma this relationship disappeared (nonsignificant) and its directionality was reversed (Weems \& Carrion, 2007). Low morning cortisol levels were also associated with increased PTSD symptoms in an Armenian earthquake study (Goenjian et al., 1996). The very few studies of parentally bereaved children that have examined links between cortisol and psychiatric symptoms show a similar hypocortisolism response. A greater number of negative life events following the loss of a parent predicted lower cortisol levels (measured before, during, and after a conflict discussion task with the surviving parent) 7 years later (Hagan, Luecken, Sandler, $\&$ Tein, 2010). In a retrospective study of college students, early loss experience was associated with decreased salivary cortisol response to awakening (Meinlschmidt \& Heim, 2005).

Inconsistencies in the extant literature are not surprising given that published studies have examined very different types of stressors (e.g., natural disasters, abuse, parental loss) - in samples that have differed in age, gender, time since trauma and duration of trauma - and have used differing measures of HPA axis function (e.g., mean cortisol levels, cortisol awakening response, diurnal decline, challenge reactivity; Adam \& Kumari, 2009; Gunnar \& Vasquez, 2001). The ability to obtain cortisol data noninvasively has made studies in children much more feasible, but there has been a tendency to treat disparate HPA axis measures equivalently and to regard "hyper" or "hypo" activity simplistically. Extensive use of salivary cortisol measures in epidemiological work has increasingly focused on the cortisol awakening response (CAR) and diurnal decline, which can be efficiently obtained by sampling upon awakening, 30 minutes later, and in the evening (Adam \& Kumari, 2009). The normal circadian rhythm of cortisol produces rising levels in the early morning hours, a further abrupt rise that commonly leads to the highest levels of the day 30 to 45 minutes after awakening, and falling levels through the rest of the day to an evening nadir. The CAR, calculated as the magnitude of rise from awakening to 30 minutes thereafter, appears to be influenced by different regulatory processes than the remaining diurnal profile (Rosmalen et al., 2005), and it likely provides different information about the stress system than do other measures of HPA reactivity. We chose to focus our analyses on the CAR because it has been the component of cortisol's circadian rhythm most extensively examined in psychosocial stress studies, and because it has been standardized in a way that facilitates comparison across studies.

Despite variation in methods and results, a growing body of evidence supports the idea that reduced stress reactivity may be associated with more maladaptive outcomes. Hypocortisolism has been linked in adults with avoidant coping strategies (Tull, Sheu, Butler, \& Cornelious, 2005) and a history of parental psychopathology (Luecken \& Appelhans, 2006). In trauma-exposed children (including those exposed to parental loss), avoidant coping and/or emotional suppression (Kaplow, Dodge, Amaya-Jackson, \& Saxe, 2005; Kaplow, Gipson, Horwitz, Burch, \& King, in press; Shapiro, Kaplow, Dodge, \& Amaya-Jackson, 2012) as well as poor functioning of the surviving caregiver (Lin, Sandler, Ayers, Wolchik, \& Luecken, 2004) predict psychological distress. Each likely factors into the relation between grief and HPA axis activity, but these complex relations have yet to be examined in parentally bereaved youth.

This study examined bereaved children's HPA-axis functioning, as measured by the CAR, in relation to psychological distress, coping, and the surviving parent's grief response. We hypothesized that greater child psychiatric symptoms, child avoidant coping, and parental psychopathology would each be associated with a dampening of child CAR. To our knowledge, this is the first study to focus specifically on the CAR as a measure of HPA axis functioning in relation to both psychological and environmental factors in parentally bereaved children following recent parental death.

\section{Method}

\section{Participants and Procedures}

Participants were 38 children ( 20 female) with recent parental loss (previous 6 months) and 28 of their surviving caregivers 
Table 1

Descriptive Data for Variables of Interest

\begin{tabular}{|c|c|c|}
\hline Variable & $M$ & $S D$ \\
\hline Day 1 CAR & 0.11 & 0.22 \\
\hline Day 2 CAR & 0.09 & 0.21 \\
\hline Day 3 CAR & 0.06 & 0.22 \\
\hline Depressive symptoms & 6.41 & 4.66 \\
\hline Maladaptive grief & 59.57 & 27.98 \\
\hline Anxiety symptoms & 51.99 & 21.36 \\
\hline Posttraumatic stress symptoms & 24.71 & 15.33 \\
\hline Avoidant coping & 17.67 & 10.71 \\
\hline Life stress & 9.02 & 4.66 \\
\hline Maladaptive grief (parent) ${ }^{\mathrm{a}}$ & 25.95 & 8.58 \\
\hline
\end{tabular}

Note. $N=38 . \mathrm{CAR}=$ cortisol awakening response.

${ }^{\mathrm{a}} n=28$.

(23 females). All surviving caregivers were either biological or adoptive parents, and all children lost either their biological or adoptive parent. Twenty-eight unique families participated; five of these had multiple children participating in the study. Children were aged $7-12$ years $(M=9.6 ; S D=2.04), 71 \%$ White, $13 \%$ Black, $11 \%$ Asian American, and 6\% Hispanic or other, and were recruited through bereavement support centers and advertisements in three cities in Michigan.

Children's surviving caregivers were, on average 42.76 years old $(S D=8.33)$; the sample of surviving parents had a similar racial composition to the sample of children. A mean of 75.29 days $(S D=47.10)$ had elapsed since the parent's death. Most deceased parents died of a sudden (40\%) or anticipated (34\%) natural cause (i.e., disease); fewer died from an accident (13\%) or suicide (13\%).

Assessments involved self-report instruments and in-person, semistructured interviews (conducted separately by trained interviewers with parents and children). Parents provided information about their own mental health as well as their child's mental health, descriptive details of the loss, the family's broader circumstances and functioning, and demographics. Children provided information about their own thoughts and feelings about the death, mental health, coping, and family functioning. At the conclusion of the interview, each parentchild dyad participated in an interaction task in which they responded to two prompts designed to elicit positive memories about the deceased parent and generate conversation between parents and children. Communication efficacy was scored using a behavioral coding protocol; scores were retained for use in a separate study of parent-child communication following loss (see Shapiro, Howell, \& Kaplow, in press). Families were compensated monetarily.

Participants were asked to provide saliva samples in their homes at three different time points (upon awakening, 30 minutes later, and in the evening) over the course of 3 days, beginning the day after the interview. Saliva was collected using
Salivette sampling tubes (Sarstedt, Nümbrecht, Germany) and samples were frozen (in home freezers and then at $-80^{\circ} \mathrm{C}$ in the lab) until thawed, centrifuged ( $15 \mathrm{~min}, 3000 \mathrm{~g}$, and $4^{\circ} \mathrm{C}$ ), and the supernatants used for assay. Cortisol levels were determined by enzyme immunoassay (EIA, Salimetrics, State College, PA) and each sample was assayed in duplicate. The sensitivity of the assay was $.003 \mu \mathrm{g} / \mathrm{dL}$. The intra-assay coefficient was $9.13 \%$ and $6.25 \%$ for low and high hormone, respectively. The interassay coefficient for low and high hormone was $11.63 \%$ and $7.87 \%$. The CAR was calculated as cortisol rise from awakening to 30 minutes later.

\section{Measures}

Life stress was assessed using the Negative Life Events Scale (NLES; Sandler et al., 1992), consisting of 36 items. Caregivers reported (yes/no) whether their child had experienced death-related adversities (e.g., "other kids teased your child because of his/her parent's death") or general stressful life events (e.g., "you lost your job") since the death, with yes responses summed to create a total score. Summed scores ranged from $4-18$ adversities $(M=9.26 ; S D=4.51)$.

Child anxiety was measured using the Multidimensional Anxiety Scale for Children, a self-report measure of generalized anxiety (MASC; March, Parker, Sullivan, Stalings, \& Conners, 1997). The MASC includes 39 items (e.g., "I try to stay near my mom or dad"), which are rated on a 4-point scale from $1=$ Never true to $4=$ Often true $(\alpha=.92)$. Scores ranged from 16 to $91(M=50.21 ; S D=20.10)$. A score of 65 or above typically represents clinically significant anxiety symptoms. Nine children $(21 \%)$ met or exceeded this cutoff.

Child depression was assessed using the Short Mood and Feelings Questionnaire (SMFQ; Angold et al., 1995). The SMFQ is a 13-item self-report measure of depressive symptoms within the past 2 weeks $(\alpha=.79)$. Items (e.g., "I felt miserable or unhappy") are rated on a 3-point scale from $1=$ Not true to $3=$ True; summed scores ranged from 0 to 19 $(M=6.15 ; S D=4.53)$. A score of 8 or higher typically represents clinically significant symptoms; 13 children (31\%) met or exceeded this cutoff.

Posttraumatic stress symptoms (PTSS) were assessed with the UCLA PTSD Reaction Index (Steinberg, Brymer, Decker, \& Pynoos, 2004). Items (e.g., "When something reminds me of what happened I get very upset, afraid or sad") are rated on a 5-point scale from $0=$ Never happens to $4=$ Happens most of the time. The total score was the sum of ratings for 17 symptoms $(\alpha=.91)$; summed scores ranged from 0 to 55 $(M=24.71 ; S D=15.33)$. A score of 38 or higher represents clinically significant symptoms of PTSD; five children (14\%) met or exceeded this cutoff.

Child maladaptive grief was assessed with the Inventory of Complicated Grief-Revised (ICG-R; Melhem, Moritz, Walker, Shear, \& Brent, 2007). The ICG-R is a measure of maladaptive grief, modified for use with children, containing 36 items 
(e.g., "I feel like the future has no meaning or purpose without my mom/dad"), which are rated on a 5-point scale from $1=$ Never to $5=A$ lot $(\alpha=.93)$. Summed scores ranged from 14 to $115(M=55.90 ; S D=28.83)$. Clinical cutoff scores have yet to be empirically derived for this measure in samples of bereaved children.

Avoidant coping was assessed with the Active Inhibition Scale (Ayers, Sandler, \& Twohey, 1998), an inventory of avoidant coping behaviors developed for use with bereaved youth. Eleven items (e.g., "When you've felt sad, you tried not to let anybody know") are rated on a 5-point scale from $1=$ Never to $5=A$ lot $(\alpha=.88)$. Summed scores ranged from 1 to $42(M=17.75 ; S D=10.65)$.

Parent maladaptive grief was assessed with the PG-13, based on proposed diagnostic criteria for Prolonged Grief Disorder (Prigerson et al., 2009). Twelve items (e.g., "Since the time of your loss, how often have you felt yourself longing or yearning for the person who died") are rated on a 5-point scale from $1=$ Not at all to $5=$ Several times a day and are summed to create a maladaptive grief score $(\alpha=.87)$. Maladaptive grief scores ranged from 12 to $48(M=25.65 ; S D=8.70)$. A score of 25 or higher represents clinically significant symptoms of complicated grief; 28 surviving parents $(46 \%)$ met or exceeded this cutoff.

\section{Data Analysis}

Bivariate analyses used Pearson's correlations and mean differences assessed via $t$ tests. Due to concerns about our small sample size, statistical power, and risk of a Type I error when conducting multiple comparsions, further analyses examined associations among variables of interest and Day 1 CAR only.

Five participating families had multiple children in the study, suggesting a potential confounding effect of nonindependent data. To correct for this possibility, all further analyses were conducted using linear mixed modeling, which accounts for potential correlations among responses provided by children in the same family. Linear mixed models assessed associations between Day 1 child CAR and (a) each of the symptom domains (child posttraumatic stress, anxiety, maladaptive grief, and depression); (b) child avoidant coping; and (c) parent maladaptive grief.

\section{Results}

Descriptive data for cortisol and other variables are presented in Table 1. Pearson's correlations and $t$ tests showed that race, age, life stress, the cause of death, and length of time since loss were not associated with our primary variables of interest, so we did not control for them in further analyses. Although girls exhibited higher levels of PTSD symptoms than boys, $t(33)=-2.35, p=.025$, no gender differences were noted among any of the other variables. Bivariate analyses showed very little association between children's Day 2 or Day 3 CAR and symptom domains of interest.

Results (see Table 2) showed significant relations between a dampening of child CAR (reduction in slope between a.m. wake-up and 30 minutes later) and more symptoms of child posttraumatic stress, $F(1$, $22.6)=7.10, p<.05$; anxiety, $F(1,25.19)=6.26, p<.05$; maladaptive grief, $F(1,22.67)=7.07, p<.05$; and depression, $F(1,25.92)=4.93, p<.05$. A dampening of CAR was also associated with children's greater use of avoidant coping, $F(1$, $22.47)=10.06, p<.01$, as well as more parent maladaptive grief symptoms, $F(1,26.13)=9.39, p<.01$.

Post hoc analyses searched for links between psychological measures and absolute levels of cortisol at awakening, 30 minutes later, and in the evening. Even using loose criteria (by not correcting for multiple comparisons), none of these correlations were significant, except for a few with the +30 minute sample, which were redundant with the CAR associations because this also captures the response to awakening.

Table 2

Correlations Between Variables of Interest

$\begin{array}{lllllllll}1 . & 2 . & 3 . & 4 . & 5 . & 6 . & 7 . & 8 .\end{array}$

1. Day 1 CAR

2. Day 2 CAR

3. Day 3 CAR

4. Anxiety

5. Posttraumatic stress

6. Avoidant coping

7. Depression

8. Maladaptive grief

9. Maladaptive grief (parent) ${ }^{\mathrm{a}}$

$\begin{array}{ccc}- & & \\ .02 & - & \\ .32 & .28 & - \\ -.45^{*} & -.27 & -.14 \\ -.45^{*} & -.31 & -.01 \\ -.53^{* *} & -.36 & -.21 \\ -.40^{*} & -.33 & .02 \\ -.47^{* *} & -.33 & .32 \\ -.43^{*} & .19 & -.38^{*}\end{array}$

Note. $N=38 . \mathrm{CAR}=$ cortisol awakening response.

${ }^{\mathrm{a}} n=28$.

${ }^{*} p \leq .05 .{ }^{* *} p \leq .01{ }^{* * *} p \leq .001$. 


\section{Discussion}

Findings support links between avoidant coping, child psychological distress, parental maladaptive grief, and a dampened CAR in parentally bereaved children. These findings are consistent with other studies that have viewed hypocortisolism as a marker of maladaptation. Hypocortisolism can reflect allostatic load (Hellhammer \& Wade, 1993; McEwen 1998) and appears to develop in some youth following prolonged periods of elevated cortisol in response to trauma or extended periods of general life stress. Given that the death of a parent is not an isolated incident (e.g., parental loss often occurs in the context of other significant life stressors; Kaplow et al., 2010), may have been anticipated for months or years (i.e., $34 \%$ of our sample experienced an anticipated death), and is usually followed by numerous secondary adversities (Hagan, Luecken, Sandler, \& Tein, 2010), the current study's findings may indeed support the idea that accumulating allostatic load can lead to blunted HPA axis reactivity, at least as reflected in the CAR.

Follow-up, longitudinal work is needed to test the hypothesis that accumulated burdens of early trauma/stress can alter set points in stress response systems that contribute to failures in normal system activation and subsequent development of psychiatric symptoms. There are numerous potential pathways through which this could occur including compromised parentchild communication, which is often identified in stressed and/or depressed mothers (Shapiro et al., in press; Yehuda, Halligan, \& Bierer, 2001), predicts both parents' (Granger et al., 1998) and children's cortisol levels (Smeekens, RiksenWalraven, \& van Bakel, 2007), and may be more likely to occur when individuals employ avoidant coping strategies. Our own prior work suggests that in trauma-exposed children (including those exposed to parental loss), avoidant coping predicts psychological distress (Kaplow et al., 2005, in press; Shapiro et al., 2012). Here we show that avoidant coping also predicts a blunted CAR. Others have demonstrated that poor functioning of the surviving caregiver (Lin et al., 2004) predicts psychological distress in the child, and here we show that this also predicts blunted child CAR. The causal path may lead from parenting factors that contribute to poor coping with loss within some families (e.g., avoidant coping responses, poor communication) and together contribute to accumulating allostatic load and reduced HPA axis reactivity. Our data, however, cannot tell us if the altered CAR is a biomarker of pre existing vulnerability, or reflects a mediating pathway from early life stress exposure to psychiatric symptom expression. Thus, larger, longitudinal studies are needed.

Another explanation for blunted CAR is suggested by our finding that the associations detected were consistent across measures for Day 1 CAR, but largely disappeared for Day 2 and Day 3 CAR. Much of the literature has framed HPA axis biomarkers as predictors of clinical outcomes, but our results raise the possibility that blunted CAR seen in the more troubled children was a result of stressful and emotionally challenging events on the interview day. Interviews asked children to reveal thoughts and feelings about the deceased parent. Although not an explicit part of the current study, the assessment concluded with a parent-child interaction task during which they were asked to talk together about positive memories of the deceased as well as characteristics that the child may have had in common with the deceased parent. How could these events or their subsequent processing within the family (or lack thereof) impact next-day CAR and not have a similar impact on Day 2 and Day 3 CAR?

The CAR may primarily reflect adrenal responsivity (Schmidt-Reinwald et al., 1999) rather than more central aspects of HPA regulatory control, and may be shaped by both neural processes associated with the awakening cycle (Clow, Hucklebridge, Stalder, Evans, \& Thorn, 2010) and psychological processes occurring during sleep (Born, Hansen, Marshall, Molle, \& Fehm, 1999). Evidence suggests that CAR may be most strongly shaped by state factors (Armario,Valles, Dal-Zotto, Marquez, \& Belda, 2004; Stalder, Evans, Hucklebridge, \& Clow, 2010). A recent review of CAR neurobiology (Clow et al., 2010) highlights its inconsistent relationship with psychosocial and health variables, emphasizes the role of extrahypothalamic factors in shaping its cortisol rise, and suggests its most critical role may be in the transition from sleep to wakefulness, with significant regulation by the suprachiasmatic nucleus through neural modulation of adrenal sensitivity to adrenocorticotropic hormone. This response appears to be carefully orchestrated by interacting systems managing the transition from sleep. Consistent with this view, chaotic or unpredictable sleep/wake cycles have been associated with blunted daytime cortisol pattern for some populations of children (Gunnar \& Vasquez, 2001).

It is possible that the content of the interview itself, especially the parent-child interaction in which discussions regarding memories of the deceased person were the focus, induced distress primarily for those children exhibiting avoidant coping patterns and experiencing parental maladaptive grief, which may have contributed to disrupted sleep and subsequent blunting of the next-day CAR. Behavioral coding of the dyadic parent-child interviews from this sample indeed suggests that children's observed discomfort during the interaction predicts next-day CAR blunting (Shapiro, 2012), and other studies have found that parent-child discussions may produce declining cortisol patterns (Adam, Klimes-Dougan, \& Gunnar, 2007). The fact that the only significant correlate of Day 2 or 3 CAR is parental maladaptive grief is consistent with the idea that the interview itself may have served as a standardized trauma or loss reminder (Layne et al., 2006), which created more temporary disruptions in those families already struggling with maladaptive grief reactions, perhaps contributing to sleep disruption and CAR blunting.

Existing evidence strongly supports the idea that priorday emotional experiences significantly shape next-day CAR (Stalder et al., 2010). Further work is needed to determine whether this occurs secondarily through effects on sleep, or through an interaction between acute emotional experiences 
and neuroendocrine control system set points shaped by prior stress exposures. The latter is more consistent with the accumulating allostatic load hypothesis proposed earlier, wheras sleep mediation suggests a different mechanism. Both factors may be in play, and each may be more strongly reflected in different measures of HPA axis function. Further work exploring both of these possibilities must take into account the complexity of the biology involved in HPA axis functioning.

\section{Limitations}

The small, relatively homogenous sample may prohibit generalizability and the cross-sectional study design precludes causal inferences. The small sample size also may have affected our ability to detect significant results; post hoc power analyses for our hypothesized correlations identified a probability of between $59.4 \%$ and $86.3 \%$ of detecting correlations of the magnitude identified here using a sample of this size, suggesting that a larger sample might yield more stable results.

Although the duration of time since the death was not significantly associated with any of our variables of interest, other studies have noted the potentially important role of time since trauma in the relation between exposure to trauma and cortisol (Bevans et al., 2008; Weems \& Carrion, 2007). Future studies of bereaved youth that include not only the time since the death, but the duration of ongoing stressors (including possible anticipation of the death) prior to the death itself are needed to better understand how timing and duration of trauma exposure may play a role in the CAR.

Finally, the measure of childhood maladaptive grief (the Inventory of Complicated Grief-Revised; Melhem et al., 2007) was developed and validated on samples of grieving adults, and patterns of maladaptive grief are likely to differ in children (Nader \& Layne, 2009). Future studies would benefit from grief-related assessment tools grounded in developmental theory and designed to capture potential age-related manifestations of grief dimensions.

\section{Clinical Implications}

An understanding of the role of HPA psychobiology in relation to childhood bereavement can help reduce bereavement-related psychopathology and promote healthy development and adaptive resilience. Specifically, the identification of modulators of the HPA axis can guide efforts to reduce exposure to the types of bereavement-related stress that are damaging to children's psychological health. A recent study of bereaved children provides evidence that preventive interventions may mitigate the negative effects of early adversity on the neuroendocrine system. Specifically, children participating in a family-focused intervention for parentally bereaved youth exhibited higher cortisol levels (i.e., less attenuated cortisol secretions) 6 years later compared to children in the control condition (Luecken et al., 2010).

The preliminary and cross-sectional nature of the current study, as well as the relative dearth of studies examining the
CAR in relation to psychiatric symptoms in bereaved youth prevent us from reaching definitive conclusions regarding the specific clinical implications of our findings. It appears, however, that bereaved children who exhibit a dampened CAR, avoidant coping strategies, and have surviving parents who experience maladaptive grief reactions may be at increased risk for anxiety, depression, posttraumatic stress, and maladaptive grief. Gunnar and Vasquez (2001) speculate that a dampened $\mathrm{CAR}$, in addition to increasing risk for psychiatric symptoms, may also alter the child's capacity to maintain alertness during the day, and over the longer term, hypocortisolism may impact brain development, cognitive functioning, and immune system functioning. Understanding the specific biological mechanisms associated with behavioral, psychological, and physical health symptoms in bereaved youth will aid the further development and adaptation of interventions to reduce the potentially detrimental impact of unavoidable stress associated with parental death.

\section{References}

Adam, E. K., Klimes-Dougan, B., \& Gunnar, M. R. (2007). Social regulation of the adrenocortical response to stress in infants, children and adolescents: Implications for psychopathology and education. In D. Coch, G. Dawson, \& K. W. Fischer (Eds.), Human behavior, learning, and the developing brain: Atypical development (pp. 264-304). New York: Guilford Press.

Adam, E. K., \& Kumari, M. (2009). Assessing salivary cortisol in largescale, epidemiological research. Psychoneuroendocrinology, 34, 14231436. doi:10.1016/j.psyneuen.2009.06.011

Angold, A., Costello, E. J., Messer, S. C., Pickles, A., Winder, F., \& Silver, D. (1995). Development of a short questionnaire for use in epidemiological studies of depression in children and adolescents. International Journal of Methods in Psychiatric Research, 5, 237-249. doi:1049-8931/95/040237-13

Armario, A., Valles, A., Dal-Zotto, S., Marquez, C., \& Belda, X. (2004). A single exposure to severe stressors causes long-term desensitisation of the physiological response to the homotypic stressor. Stress, 7, 157-72. doi:10.1080/10253890400010721

Ayers, T. S., Sandler, I. N., \& Twohey, J. L. (1998). Conceptualization and measurement of coping in children and adolescents. In T. H. Ollendick \& R. J. Prinz (eds.). Advances in Clinical Child Psychology, Vol. 20 (pp. 243-301). New York: Plenum.

Bevans, K., Cerbone, A., \& Overstreet, S. (2008). Relations between recurrent trauma exposure and recent life stress and salivary cortisol among children. Development and Psychopathology, 20, 257-272. doi:10.1017/S0954579408000126

Born, J., Hansen, K., Marshall, L., Molle, M., \& Fehm, H. (1999). Timing the end of nocturnal sleep. Nature, 397, 29-30. doi:10.1038/16166

Carlson, M., \& Earls, F. (1997). Psychological and neuro-endocrinological sequelae of early social deprivation in institutionalized children in Romania. Annals of the New York Academy of Sciences, 807, 419-428. doi:10.1111/j.1749-6632.1997.tb51936.x

Clark, D. C., Pynoos, R. S., \& Goebel, A. E. (1994). Mechanisms and processes of adolescent bereavement. New York: Cambridge University Press.

Clow, A., Hucklebridge, F., Stalder, T., Evans, P., \& Thorn, L. (2010) The cortisol awakening response: More than a measure of HPA axis function. Neuroscience and Biobehavioral Reviews, 35, 97-103. doi:10.1016/j.neubiorev.2009.12.011 
Dowdney, L. (2000). Annotation: Childhood bereavement following parental death. Journal of Child Psychology and Psychiatry, 41, 819-830. doi:10.1111/1469-7610.00670

Goenjian, A. K., Yehuda, R., Pynoos, R. S., Steinberg, A. M., Tashjian, M., Yang, R. K., et al. (1996). Basal cortisol and dexamethasone suppression of cortisol and MHPG among adolescents after the 1988 earthquake in Armenia. American Journal of Psychiatry, 153, 929-934.

Goenjian, A. K., Walling, D., Steinberg, A. M., Roussos, A., Goenjian, H. A., \& Pynoos, R. S. (2009). Depression and PTSD symptoms among bereaved adolescents $61 / 2$ years after the 1988 Spitak earthquake. Journal of Affective Disorders, 112, 81-84. doi:10.1016/j.jad.2008.04.006

Gold, P. W., Goodwin, F. K., \& Chrousos, G. P. (1988). Clinical and biochemical manifestations of depression: Relations to the neurobiology of stress. New England Journal of Medicine, 319, 348-353. doi:10.1056/NEJM198808113190606

Granger, D. A., Serbin, L. A., Schwartzman, A., Lehoux, P., Cooperman, J., \& Ikeda, S. (1998). Children's salivary cortisol, internalising behaviour problems, and family environment: Results from the Concordia Longitudinal Risk Project. International Journal of Behavioral Development, 22, 707-728 doi:10.1080/016502598384135

Gunnar, M. R., \& Vazquez, D. M. (2001). Low cortisol and a flattening of the expected daytime rhythm: Potential indices of risk in human development. Development and Psychopathology, 13, 516-538. doi:10.1017/S0954579401003066

Hagan, M. J., Luecken, L. J., Sandler, I. N., \& Tein, J. (2010). Prospective effects of post-bereavement negative events on cortisol activity in parentally bereaved youth. Developmental Psychobiology, 52, 394-400. doi: 10.1002/dev.20433

Heim, C., Ehlert, U., \& Hellhammer, D. H. (2000). The potential role of hypocortisolism in the pathophysiology of stress-related bodily disorders. Psychoneuroendocrinology, 25, 1-35. doi:10.1016/S0306-4530(99)00035-

Hellhammer, D., \& Wade, S. (1993). Endocrine correlates of stress vulnerability. Psychotherapy \& Psychosomatics, 60, 8-17. doi:10.1159/000288675

Kaplow, J., Dodge, K., Amaya-Jackson, L., \& Saxe, G. (2005). Pathways to PTSD Part II: Sexually abused children. American Journal of Psychiatry, 162, 1305-1310. doi:10.1176/appi.ajp.162.7.1305

Kaplow, J.B., Gipson, P., Horwitz, A., Burch, B., \& King, C. (2013). Emotional suppression mediates the relation between adverse life events and adolescent suicide: Implications for prevention. Prevention Science. Epub ahead of print. doi:10.1007/s11121-013-0367-9

Kaplow, J. B., Layne, C. M., Pynoos, R. S., Cohen, J., \& Lieberman, A. (2012). DSM-V diagnostic criteria for bereavement-related disorders in children and adolescents: Developmental considerations. Psychiatry, 75, 242-265. doi:10.1521/psyc.2012.75.3.243

Kaplow, J. B., Saunders, J., Angold, A., \& Costello, E. J. (2010). Psychiatric symptoms in bereaved versus nonbereaved youth and young adults: A longitudinal epidemiological study. Journal of the American Academy of Child \& Adolescent Psychiatry, 49, 1145-1154. doi:10.1016/j.jaac.2010.08.004

Kaufman, J., Birmaher, B., Perel, J., Dahl, R. E., Moreci, P., Nelson, B., ...., \& Ryan, N. (1997). The corticotropin-releasing hormone challenge in depressed abused, depressed nonabused, and normal control children. Biological Psychiatry, 42, 669-679. doi:10.1016/S0006-3223(96)00470-2

Layne, C. M., Warren, J. S., Saltzman, W. R., Fulton, J. B., Steinberg, A. M., \& Pynoos, R. S. (2006). Contextual influences on posttraumatic adjustment: Retraumatization and the roles of revictimization, posttraumatic adversities and distressing reminders. In L. A. Schein, H. I. Spitz, G. M. Burlingame, P. R. Muskin \& S. Vargo (Eds.), Psychological effects of catastrophic disasters. Group approaches to treatment (pp. 235-286). New York: Haworth Press.
Lin, K. K., Sandler, I. N., Ayers, T. S., Wolchik, S. A., \& Luecken, L. J. (2004). Resilience in parentally bereaved children and adolescents seeking preventive services. Journal of Clinical Child and Adolescent Psychology, 33, 673-683. doi:10.1207/s15374424jccp3304_3

Luecken, L. J., \& Appelhans, B. M. (2006). Early parental loss and salivary cortisol in young adulthood: The moderating role of family environment. Development and Psychopathology, 18, 295-308. doi:10.1017/S0954579406060160

Luecken, L. J., Hagan, M. J., Sandler, I. N., Tein, J., Ayers, T., \& Wolchik, S. (2010). Cortisol levels six years after participation in the Family Bereavement Program. Psychoneuroendocrinology, 35, 785-789. doi:10.1016/j.psyneuen.2009.11.002

March, J. S., Parker, J. D. A., Sullivan, K., Stalings, P., \& Conners, C. K. (1997). The Multidimensional Anxiety Scale for Children (MASC): Factor structure, reliability, and validity. Journal of the American Academy of Child and Adolescent Psychiatry, 36, 554-565. doi:10.1097/00004583199704000-00019

McEwen, B. (1998). Protective and damaging effects of stress mediators. New England Journal of Medicine, 338, 171-179. doi:10.1056/ NEJM199801153380307

Meinlschmidt, G., \& Heim, C. (2005). Decreased cortisol awakening response after early loss experience. Psychoneuroendocrinology, 30, 568-576. doi:10.1016/j.psyneuen.2005.01.006

Melhem, N. M., Moritz, G., Walker, M., Shear, M. K., \& Brent, D. (2007). Phenomenology and correlates of complicated grief in children and adolescents. Journal of the American Academy of Child and Adolescent Psychiatry, 46, 493-499. doi:10.1097/chi.0b013e31803062a9

Melhem, N. M., Porta, G., Shamseddeen, W., Walker Payne, M., \& Brent, D. A. (2011). Grief in children and adolescents bereaved by sudden parental death. Archives of General Psychiatry, 68, 911-919. doi:10.1001/archgenpsychiatry.2011.101

Nader, K. O., \& Layne, C. M. (2009). Maladaptive grieving in children and adolescents: Discovering developmentally-linked differences in the manifestation of grief. Stress Points, 23, 12-15.

Pfeffer, C. R., Altemus, M., Heo, M., \& Jiang, H. (2007). Salivary cortisol and psychopathology in children bereaved by the September 11, 2001 terror attacks. Biological Psychiatry, 61, 957-965. doi:10.1016/j.biopsych.2006.07.037

Prigerson, H. G., Horowitz, M. J., Jacobs, S. C., Parkes, C. M., Aslan, M., Goodkin, K., ... Maciejewski, P. K. (2009). Prolonged grief disorder: Psychometric validation of criteria proposed for DSM-V and ICD11. Public Library of Science, 6, e1000121. doi:10.1371/journal.pmed .1000121

Rao, U., Dahl, R. E., Ryan, N. D., Birmaher, B., Williamson, D. E., Giles, D. E., Rao, R., Kaufman, J., \& Nelson, B. (1996). The relationship between longitudinal clinical course and sleep and cortisol changes in adolescent depression. Biological Psychiatry, 40, 474-484. doi:10.1016/0006-3223(95) 00481-5

Rosmalen, J. G. M., Oldehinkel, A. J., Ormel, J., de Winter, A. F., Buitelaar, J. K., \& Verhulst, F. C. (2005). Determinants of salivary cortisol levels in 10-12-year-old children; a population-based study of individual differences. Psychoneuroendocrinology, 30, 483-495. doi:10.1016/j.psyneuen.2004.12.007

Sandler, I. N., West, S. G., Baca, L., Pillow, D. R., Gersten, J., Rogosch, F., . . . Ramirez, R. (1992). Linking empirically based theory and evaluation: The Family Bereavement Program. American Journal of Community Psychology, 20, 491-521. doi:10.1007/BF00937756

Schmidt-Reinwald, A., Pruessner, J. C., Hellhammer, D. H., Federenko, I., Rohleder, N., Schurmeyer, T. H., \& Kirschbaum, C. (1999). The 
cortisol response to awakening in relation to different challenge tests and a 12-hour cortisol rhythm. Life Science, 64, 1653-1660. doi:10.1016/S00243205(99)00103-4

Shapiro, D. N. (2012). Psychological outcomes for both mothers and children in nontraditional families: The buffering roles of parenting practices and social support. (Unpublished doctoral dissertation, University of Michigan, Ann Arbor, MI.)

Shapiro, D. N., Howell, K. H., \& Kaplow, J. B. (in press). Associations among mother-child communication quality, childhood maladaptive grief, and depressive symptoms. Death Studies.

Shapiro, D. N., Kaplow, J. B., Dodge, K. A., \& Amaya-Jackson, L. (2012). Behavioral markers of coping and psychiatric symptoms among sexually abused children. Journal of Traumatic Stress, 25, 157-163. doi: $10.1002 /$ jts. 21674

Smeekens, S., Riksen-Walraven, J. M. A., \& van Bakel, H. J. A. (2007). Cortisol reactions in five-year-olds to parent-child interactions: The moderating role of ego-resiliency. Journal of Child Psychology and Psychiatry, 48, 649-656. doi:10.1111/j.1469-7610.2007.01753.x

Spangler, G., \& Grossmann, K. E. (1993). Biobehavioral organization in securely and insecurely attached infants. Child Development, 64, 1439-1450. doi: $10.2307 / 1131544$

Stalder, T., Evans, P., Hucklebridge, F., \& Clow, A. (2010). Associations between psychosocial state variables and the cortisol awakening re- sponse in a single case study. Psychoneuroendocrinology, 35, 209-214. doi:10.1016/j.psyneuen.2009.06.006

Steinberg, A. M., Brymer, M. J., Decker, K. B., \& Pynoos, R. S. (2004). The University of California at Los Angeles Post-traumatic Stress Disorder Reaction Index. Current Psychiatry Reports, 6, 96-100. doi:10.1007/s11920004-0048-2

Tull, E. S., Sheu, Y. T., Butler, C., \& Cornelious, K. (2005). Relationships between perceived stress, coping behavior, and cortisol secretion in women with high and low levels of internalized racism. Journal of the National Medical Association, 97, 206-212.

UNAIDS, UNICEF, \& USAID. (2004). Children on the Brink 2004: A Joint Report on New Orphan Estimates and a Framework for Action. New York: Author.

Weems, C. F., \& Carrion, V. G. (2007). The association between PTSD symptoms and salivary cortisol in youth: The role of time since trauma. Journal of Traumatic Stress, 20, 903-907. doi:10.1002/jts.20251

Yehuda, R., Halligan, S., \& Bierer, L. (2001). Relationship of parental trauma exposure and PTSD to PTSD, depressive, and anxiety disorders in offspring. Journal of Psychiatric Research, 35, 261-270. doi:10.1016/S00223956(01)00032-2

Young, E. A., Haskett, R. F., Murphy-Weinberg, V., Watson, S. J., \& Akil, H. (1991). Loss of glucocorticoid fast feedback in depression. Archives of General Psychiatry, 48, 693-699. doi:10.1001/archpsyc.1991.01810320017003 